\title{
Impact of the new Sendai framework for disaster risk reduction on Paris flood prevention program
}

\author{
Regis THEPOT, Pascal GOUJARD, Frédéric GACHE \\ EPTB Seine Grands Lacs
}

\begin{abstract}
The greater Paris region faces a significant risk of flooding due to potential spill-over from the Seine and the Marne. Because the last major flood occurred in 1910, the event has faded in the collective memory. Consequently, the population and the public authorities have difficulty imagining that such a catastrophe might repeat itself. In parallel, widespread urban expansion into flood zones has considerably aggravated the foreseeable damage if an event of a comparable intensity were to hit the region.In response to this situation, the EPTB Seine Grands Lacs a public territorial basin establishment- decided to take action to reduce this risk.It began by commissioning a study from the OECD on flood risk prevention in the Seine Basin. This study was presented in January 2014 and highlighted the considerable risk of flooding in or near Paris, which could, affect a total of nearly 5 million people, cause up to $€ 30$ billion in direct damage and affect up to 400.000 jobs. It also put forward 14 recommendations that are being implemented by the public authorities, at either the national, basin or local level.The EPTB launched in partnership with the government a second initiative for which it steers and coordinates a coherent, balanced, relevant and gradual programme of 78 flood prevention actions. As a new post-2015 framework for disaster risk reduction was adopted in Sendai in March 2015 taking in account lessons learned during the 2005-2015 period, gaps identified and future challenges, this paper addresses the question of the impact of this new international framework on the implementation of the flood prevention of Paris region. One of the main points developed is the necessity to increase public awareness, to enhance disaster preparedness for effective response and to "build back better" in recovery rehabilitation and reconstruction.
\end{abstract}

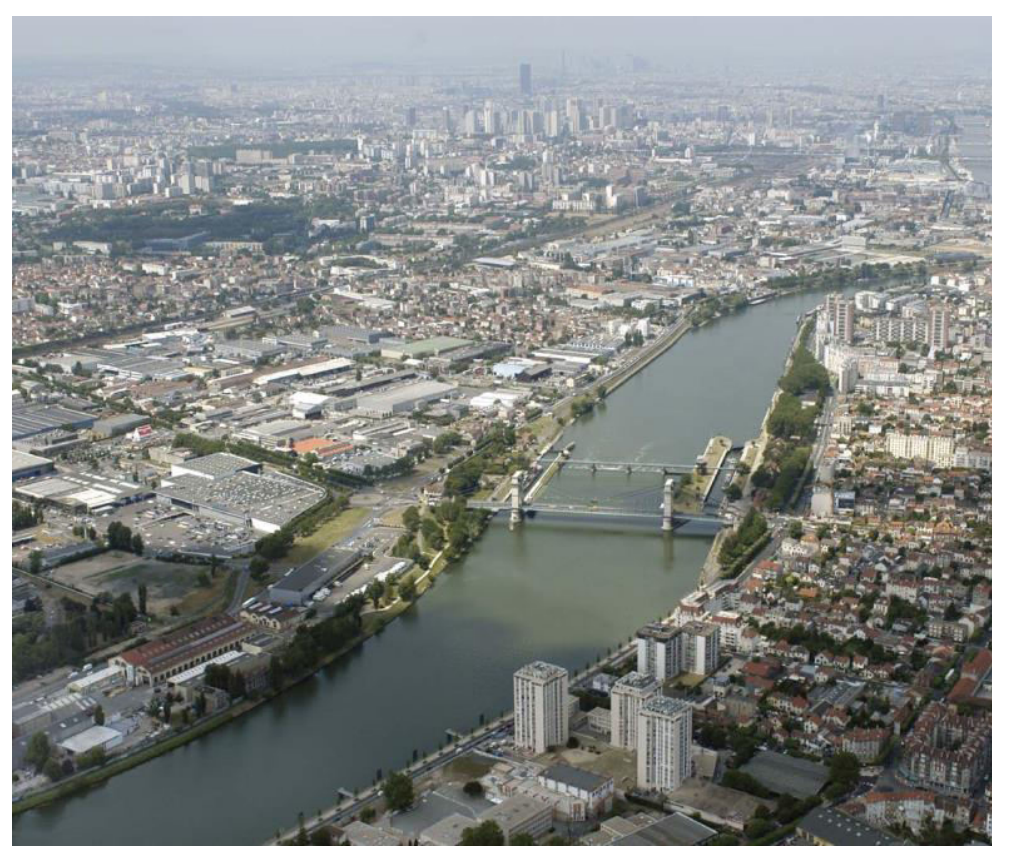

\footnotetext{
$\overline{{ }^{\mathrm{a}} \text { Corresponding author: eptb@seinegrandslacs.fr }}$
} 


\section{A major flood of the Seine river should be a huge challenge for public policy}

While the possibility of a major flood of the Seine river may initially seem to be remote, it comes back periodically to attract attention: hence, in the spring of 2013, floods upstream of the Seine basin aroused awareness in this regard. Even though it did not cause any major damage, nevertheless, this flood reopened the question of risk management and the region's vulnerability to flooding. The prospect of a historic event is a key concern for the French risk management stakeholders. The 1910 flood was particularly destructive in the context of an era marked by industrial and technological progress.

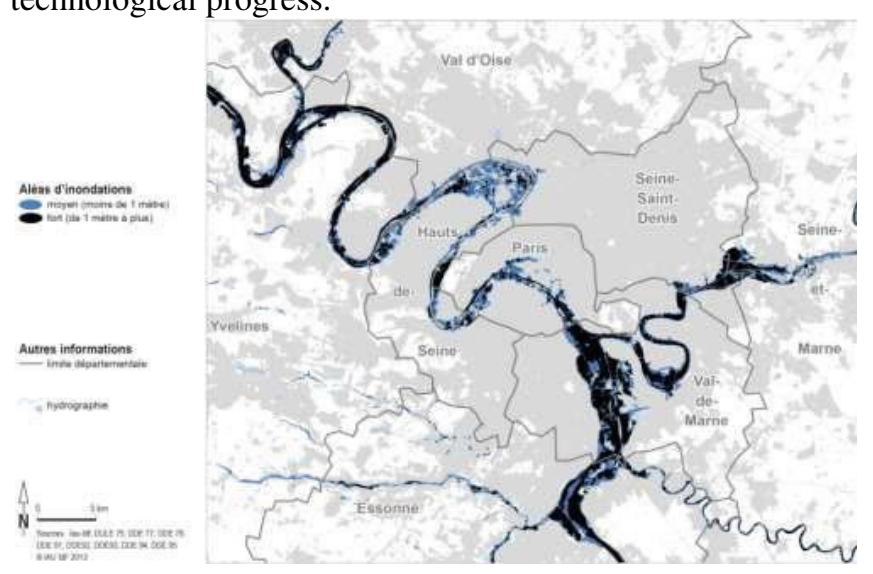

Figure 1. Map of the floodplain. Source : IAU Ile-de-France

Since that period the Ile-de-France region developped a lot and represents nowadays about one third of economic activity in France, the second largest economy in the euro area. The seat of the government and major industries are located there, as well as the main decision-making and research centres.

The 2014 OECD (Organisation for Economic Cooperation and Development) review about Seine basin Ile de France demonstrated that in the most extreme case, a Seine flood in the Ile-de-France would have direct and indirect impacts on almost 5 million people and a large number of companies, with significant economic, human and social effects. Electricity distribution would be substantially affected with almost one quarter of power sub-station flooded or cut off as a precaution and more than 1.5 million customers who could experience power cuts. Public transport could be affected with almost $140 \mathrm{~km}$ out of $250 \mathrm{~km}$ of the underground network closed as a precaution. The road network could be blocked at many points: the bridges crossing the Seine closed to traffic, due to their weakened structure, would make it impossible to travel from the right to the left bank. The drinking water supply could be interrupted around Paris where more than 5 million subscribers could suffer extended water cuts and 1.3 million a deterioration in quality.

A major flood of the Seine river should be consequently a huge challenge for public policy and the
OECD review gave several recommandations on that field, as on structural and non structural measures, most of them beeing implemented at this moment national, basin and local authorities.

\section{A flood prevention action programme (PAPI) for Paris and its inner ring departments}

The urbanisation of flood prone areas of Ile-deFrance has been ongoing since 1930, accelerating in particular as part of the development of new towns and the gradual urbanisation of first the inner, and then the outer, ring of Paris. This urbanisation most often took place with no consideration of the risks, nor any real assessment of the consequences of major flooding on human health, the economy, the environment and historical heritage.

Over the years, knowledge of the stakes directly exposed to flooding has become more detailed, but large gaps remain in the precise assessment of areas indirectly affected by damage to the networks. Problems on the networks can appear very early on, before the territories are completely flooded, and can be felt much further than the flood prone areas.

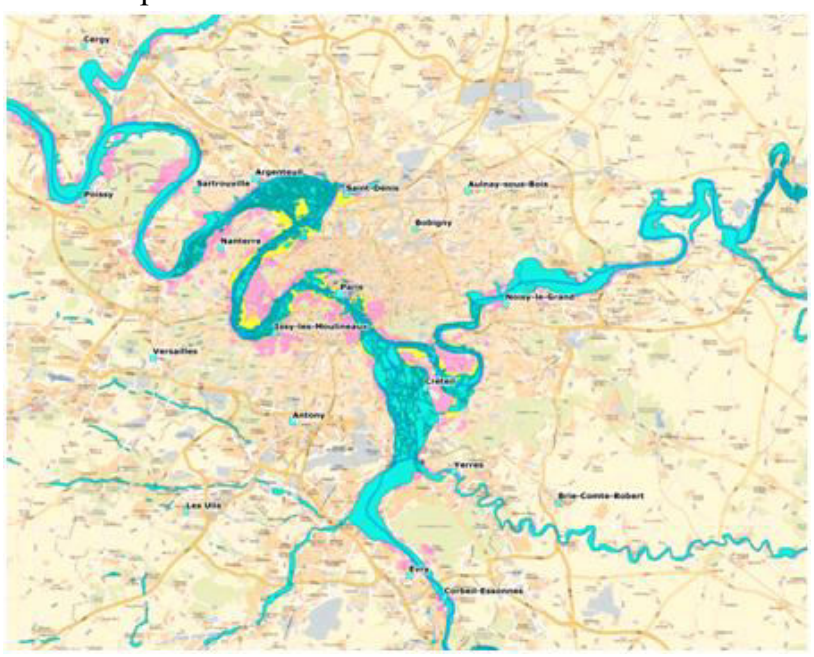

Figure 2. Map of the highest known water levels

(blue), flooded cellars (yellow) and areas with weaknesses in their electrical power networks (pink).

Source : EDF-2006

In Paris and its suburbs (400 towns and cities with 10.2 million inhabitants), human and economic activity, transport infrastructure, urban networks and equipment, and economic and political decision-making centres are all concentrated over 18,000 hectares of flood prone area. $11,000(60 \%)$ of those hectares are potentially exposed to strong to very strong hazards (more than one metre of submergence). These sectors are now two-thirds urbanised, with built-up space (dominated by housing) occupying more than $40 \%$ of the affected flood prone areas.

Of this housing in the basin upstream from the Seine and concerned by the risk of flooding, $90 \%$ is in 
Ile-de-France, namely in Paris, Hauts-de-Seine and Valde-Marne. In other words, Ile-de-France is far and away the French region with the largest number of inhabitants exposed to these hazards. According to the police prefecture, more than 850,000 people live in actual flood prone areas (were the water mark at Paris-Austerlitz to exceed $8 \mathrm{~m}$ ). All or part of the footprints of close to 56,700 establishments and 630,000 jobs, or respectively $9.5 \%$ and $11.5 \%$ of the companies and employees recorded for Ile-de-France, are potentially exposed to flooding.

Nearly 350 health establishments (public and private hospitals) are also believed to be concerned by a major event.

Several million inhabitants are potentially concerned by flooding, if account is also taken of the indirect effects of a flood on the power, sanitation, drinking water and transport networks, although a consolidated assessment of these is lacking. The power network is the one most susceptible and most essential to the Ile-de-France Region, as all the other networks are relying on that energy. It is estimated that some 1.2 million electricity consumers should potentially be affected by a major flood. Drinking water supply networks will also experience significant impacts in the event of flooding of the Seine, once the Austerlitz station hits the 6.20 metres mark. Close to 1.4 million subscribers of the Syndicat des Eaux D'Ile-de-France (SEDIF) would thus be without drinking water for a period of several days, and more than another 1.5 million would only have limited service.

On the RATP (company of public transport in Paris) network, which launched actions to reduce its subway infrastructure a decade ago, the impact of a flood would affect 140 kilometres of lines across a total of 250 $\mathrm{km}$ and would require more than a year's work to repair the concerned network, with $€ 1.5$ billion in damages.

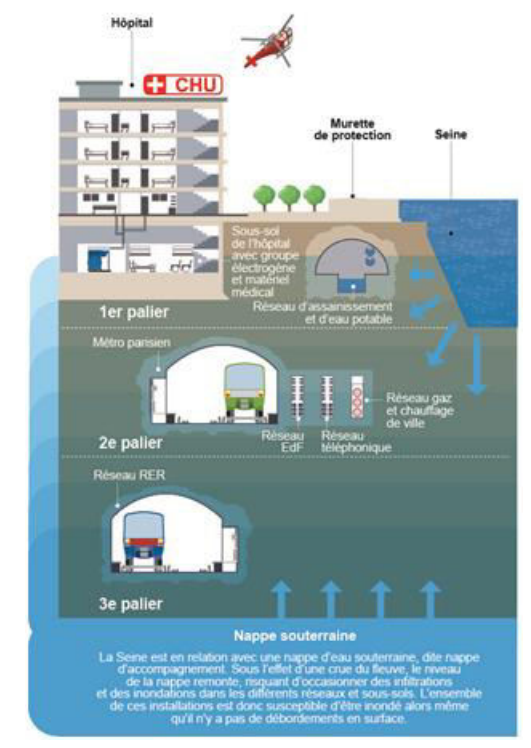

Figure 3. Network disturbances in the event of flooding of the Seine - Source: EPTB Seine Grands Lacs

\section{Facing that context and situation 68 actions were launched in 2014 in the PAPI (flood prevention action programme) programme to reach priority targets}

Inspired by the Hyogo framework for action 2005-2015 and by the national framework, the PAPI programme of actions is balanced between structural measures, acting on the hazard, and non-structural measures. Focusing mainly on the development of the culture of risk and the capacity of local public services to return to normal as quickly as possible. It must be précised that this programme is complementary of other emergency and crisis management actions directly taken in charge by the government and its representative the Préfet de police.

To increase the culture of risk amongst Ile-de-France residents and to better live with the river and its high water periods, the planned actions aim to:

- Inform and raise the awareness of all concerned audiences: inhabitants, schools, local governments (elected officials and services), public services, companies, social housing landlords and more;

- Shift land developers' urban planning and architectural practices in flood prone areas; and

- Use a wide variety of media tools: high water markers, cultural projects, videos, pamphlets, multimedia supports and more.

Stabilising the cost of damages will entail actions that aim to:

- Reduce exposure to the risk of flooding the land. In particular:

- the development of technical solutions for resilient urban planning (focusing as a priority on urban renewal);

- the development of vulnerability reduction procedures for existing property (municipal heritage including local networks, companies, etc.);

- Reinforce the system of existing protective structures, i.e.:

- the optimisation of management of the four storage reservoirs;

- conduct of a pilot district at the downstream La Bassée site;

- restoration of the barrier walls (departmental councils);

- improvement of the Seine's conveyance by pushing the wall back (Hauts-de-Seine departmental council);

- restore the Saint-Maur sector gate to operation (Val-de-Marne departmental council); and

- Further develop the detention functions of natural spaces, which requires an inventory and management of the flood expansion areas and wetlands in the Seine's upstream basin.

Reinforcing the resilience of the main public services will require shortening the time before public services 
return to satisfactory operation (in the event of a crisis), at every geographic level: regionally (for transport infrastructure, training centres, etc.), departmentally, municipally and inter-municipally (crèches, schools, PMI establishments, secondary schools, retirement homes, hospitals, homes for persons with disabilities, and so on).

The programme of action for the PAPI is estimated at an overall total of $€ 110,504,500$ excluding VAT or $€ 132,605,400$ with VAT.

The first phase (2014 to 2016) is estimated at $€ 17,134,500$ excluding VAT and the second (2017 to 2019) at $€ 93,370,000$ before VAT.

Lastly, it should be noted that the PAPI stipulates, from the outset, a midway revision in late 2016, which can be used to incorporate the provisions of the flood risk management plan for the Ile-de-France urban area and to include new actors (and especially Métropole du grand Paris as a very important new player in that field) and to adjust the forecast amounts and financing for the actions.

\section{Awareness raising actions to develop knowledge and the culture of risk}

The majority of the PAPI actions pertain to this area of intervention, with half of the 78 actions proposed by the different contracting authorities. The budget forecast for this area is $€ 6,495,200$ before VAT.

The magnitude of the planned actions, their content and their targets follow on from the diagnostic review, which showed that a large proportion of the population and of public facilities managers misunderstood how the river worked, flood hazards, the magnitude of the exposed stakes and therefore the risks incurred in Ile-de-France. The PAPI's partners have proposed a broad range of actions to raise the awareness of the different (public and private) targets deemed to be priorities: risk managers, public facilities managers and professionals. To do this, they aim to implement innovative products oriented toward new information technologies.

Some examples of actions include:

- the creation and development of a flood resource centre for the Seine's upstream basin;

- the creation, adaptation and updating of three smartphone applications;

- the installation of new high water markers;

- multiple communications processes based first on the broad array of target audiences (teaching community, population, trade unions, social housing landlords, town halls, companies, etc.) and second on the diversity of communications media planned (files, cultural projects, video, etc.), plus a graphic representation of flood prone areas in 3D augmented reality.

\section{Actions to reinforce the consideration of flood risks in land development}

This PAPI's contracting authorities made it a priority to raise awareness and train public and private developers so that they can first adapt existing constructions to flood risks and second take flood risks into better consideration in urban planning, account taken of the high levels of urban renewal in Ile-de-France and of the potential for construction in flood prone areas over the next 10 years.

As a complement to discussions of the development of flood prone areas that have already been urbanised, it was also deemed to crucial to preserve and improve the Seine's upstream flood expansion fields that are still free of any urbanisation - often referred to as "green infrastructure" - that can help to reduce flow rates along the entire system (from the stream to major rivers), at the source, while reducing water levels in downstream urbanised areas. It should be noted that the La Bassée project also pursues an environmental goal, that of rehabilitating the wetlands of major interest in downstream La Bassée, in line with the European policy of promoting green infrastructure.

The budget forecast for this area is $€ 775,000$ before VAT.

Some examples of actions include:

- $\quad$ support for the training of municipal engineers and developers, for better consideration of flood risks in land development projects (with communications documents, training cycles, etc.); and

- conduct of a study to define management measures for flood expansion fields and wetlands that contribute to flood management, to be defined through discussions launched by the DRIEE and the Seine-Normandie water agency.

\section{Actions to reduce the vulnerability of people and propert}

In view of the large quantity of public buildings built in flood prone parts of Ile-de-France (numbering in the thousands) prior to approval of special rules regarding flood risks, the reduction of their vulnerability is considered to be a priority action.

The actions proposed by the contracting authorities for this area therefore aim at a diagnostic review and the performance of adaptation work on exposed buildings, with the goal of reducing their vulnerability to flooding and, in particular, of limiting the consequences on buildings fulfilling a public service mission.

The budget forecast for this area is $€ 3,261,400$ before VAT.

Some examples of actions include:

- support for the performance of vulnerability reviews and adaptation measures for municipalities and government agencies; and work to reduce the vulnerability of government agencies. 


\section{The new Sendai framework: an opportunity for the next review of the flood prevention action programme}

\section{The Sendai framework for Disaster Risk Reduction :}

The Sendai framework for disaster risk reduction 2015-2030 was adopted at the third UN World Conference in Sendai, Japan, on March 2015. It is the outcome of stakeholders consultations initiated in March 2012 and inter-governmental negotiations from July 2014 to March 2015, supported by the United Nations Office for disaster risk reduction at the request of the UN General Assembly.

It is the successor instrument of the Hyogo framework for action (HFA).

The Sendai framework is built on elements which ensure continuity with the work done by States and other stakeholders under the HFA and introduces a number of innovations as called for during the consultations and negotiations. Many commentators have identified the most significant shifts as a strong emphasis on disaster risk management as opposed to disaster management, the definition of seven global targets, the reduction of disaster risk as an expected outcome, a goal focused on preventing new risk, reducing existing risk and strengthening resilience, as well as a set of guiding principles, including primary responsibility of states to prevent and reduce disaster risk, all-of-society and all-ofState institutions engagement. In addition, the scope of disaster risk reduction has been broadened significantly to focus on both natural and man-made hazards and related environmental, technological and biological hazards and risks. Health resilience is strongly promoted throughout.

The Sendai Framework also articulates the following: the need for improved understanding of disaster risk in all its dimensions of exposure, vulnerability and hazard characteristics; the strengthening of disaster risk governance, including national platforms; accountability for disaster risk management; preparedness to "Build Back Better"; recognition of stakeholders and their roles; mobilization of risk-sensitive investment to avoid the creation of new risk; resilience of health infrastructure, cultural heritage and work-places; strengthening of international cooperation and global partnership, and risk-informed donor policies and programs, including financial support and loans from international financial institutions.

The Sendai framework précised and defined new priorities for action:

- Understanding disaster risk.

- Strengthening disaster risk governance to manage disaster risk.

- $\quad$ Investing in disaster risk reduction for resilience.
- Enhancing disaster preparedness for effective response and to "Build Back Better" (BBB) in recovery, rehabilitation and reconstruction.

This last new priority is clearly an opportunity for the 2016 planned review of the PAPI in a context where, as the last big Seine flood occurred more than one century ago, public awareness is at a very poor level and when it is difficult to keep this kind of actions at a high level in the political agenda at a period with more and more rare public funding. Different possibilities are so currently been explored in order to adapt or add new actions tackling with that priority and some examples are presented below as "work in progress"

\section{Towards "BBB" actions in the revised PAPI}

The extreme complexity of the interdependencies between Paris networks and their high vulnerability to overflowing flood is a clear encouragement to support specific actions on that issue.

To remind, it should be noted the actions already included in the PAPI program, particularly as regards the knowledge about the vulnerability of networks:

- Animation and awareness of public services, companies or the public about the floods impacts on the networks, - Creation of resources center that will offer different documents (theses, studies, articles ...) related to the vulnerability of the Paris ;

- Awareness of equipment managers and of the public to specific vulnerabilities in Ile-de-France area; - Training of planners and technicians of public services and development of vulnerability diagnostics.

In the framework of the review of the action program, several tracks are envisaged

- Create a legal structure for sustainability in a medium and long term a project management that combines public and private actors to deal with flood risk management issues in Ile-de-France area.

- Study a computerized interface that enables the pooling of knowledge of the various operators and network managers about vulnerabilities and interdependencies for several scenarios.

- Reinforce analyses on the length of interruptions of services to the population (transport, school activities, health facilities, etc.).

- Use innovative technologies from "smart network" and the concept of "smart city" in the service of flood management.

- and last but not least, design and anticipate the terms of post flood reconstruction networks ; this approach could be applied in two complementary approaches:

The first step planned is to mobilize collective expertise driven by the CEREMA (national public Center for expertise and engineering on risks, urban and country planning, environment and mobility) in the RAITAP 
project (annexe 1) to the Paris urban networks.

\section{Conclusion}

In conclusion through the revision of the Seine river flood prevention action program (PAPI) planned at the end of 2016, it as been introduced new Sendai concepts and particularly the "Build Back Better" approach.

It seems a very stimulating and promising theme for new ideas, researches, projects and actions and particularly well adapted to basins with rare big floods.

On the Seine basin discussions are just beginning between the involved stakeholders in order to try to implement this new international concept and all the international exchanges on that new issue are welcome!

\section{References}

\section{Annexe 1 : The RAITAP project}

The RAITAP project (Rethinking preventive Action towards risk flood Including the TerritoriAl Post-flood action) is funded by the CEREMA. This project deals with a major challenge of the $21^{\text {st }}$ century to propose adaptations for more flood resilient future societies. The rising economic cost of floods coupled with demographic increase in flood areas, is a reality as highlighted by the French national strategy for flood risk management.

The disaster management cycle contains three phases:

1) the prevention during the pre-disaster time,

2) the crisis management during the disaster, then

3) the post-disaster recovery. The "pre-disaster" time and the "crisis" are both most studied phases and concentrate main financial resources and risk management tools. However, the post-disaster period is recognised to be complex, poorly understood, few anticipated, and is characterised by the implication of a wide range of stakeholders. In most cases, the collective will is to recover the initial state without learning from the disaster, and lead to an identical reconstruction of the disaster area. Nevertheless, the post-disaster period could be seen as an opportunity to better reorganize the territory to reduce its vulnerability in anticipation of future flood events.

The RAITAP study is dealing with improving the disaster responses to "build back better" in recovery phases, rehabilitation and reconstruction.

To explore this hypothesis, this work proposes to analyze the post-flood period through several feedbacks (floods in the Somme French department in 2001, flash floods in the Gard French department in 2002 and floods in Saguenay-Lac-Saint-Jean in the Canadian province of Quebec in 1996), with a special focus on temporal organization of the post-disaster management and implemented actions. These results will lead to better understanding in 1) temporal responses of various actors, financial flows involved and measures taken after the disaster, and 2) the concept of "recovery", and factors to enhance flood resilience.

Case studies on Paris urban network are going to be included in the RAITAP project.

\section{An exemple : Gennevilliers' diversion}

This project, studied by EPTB in the 90's consisted to realize a tunnel with a 10 meters diameter and a length of 13 kilometres. One of its purposes was to divert part of the flow of the Seine (until $300 \mathrm{~m} 3 / \mathrm{s}$ ) and to by-pass of a loop of the Seine in order to reduce the flow transiting upstream in Paris.

This tunnel could be also used for sanitation in order to store polluted water discharged in the loops, which is particularly sensitive due to its hydraulic system, and then to return it later at a water treatment plant downstream.

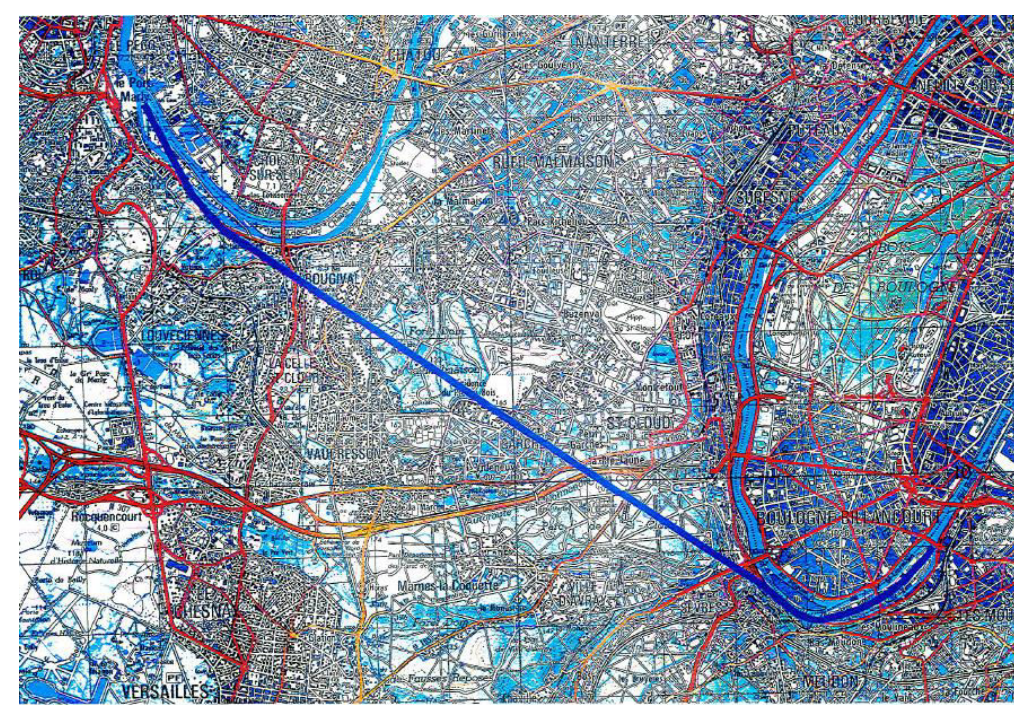

Figure 4 : Gennevilliers' diversion - tunnel - Source: EPTB Seine Grands Lacs

This expensive project asked many questions (insertion of the tunnel in the environment, socio-economical impact, speed of the flow and risk of flooding downstream, etc...) and was abandoned. It was also discussed at that time if the tunnel could be used as an expressway to make it more profitable.

It seems still impossible to realise this kind of project today. But why not to exam the interest of this Seine bypass in a BBB process and to study the feasibility of a diversion channel combined with other uses, roads, landscape and recreational areas, in a territory that would have been before flooded and devastated by a major flood of the Seine. 